\title{
Distribución espacial del sector servicios en México, 1999-2009. Especialización y diversificación desde una perspectiva macrogeográfica
}

Enrique Pérez-Campuzano. Universidad Nacional Autónoma de México, Ciudad de México, México.

Alejandro Sánchez-Zárate. El Colegio de México, Ciudad de México, México. Juan Cuadrado-Roura. Universidad de Alcalá, Madrid, Espańa.

RESUMEN | El estudio de los servicios realizado desde una perspectiva espacial sigue siendo poco frecuente en países en desarrollo. Considerando tal situación, en este artículo, tomando el caso mexicano, se muestra cómo los servicios siguen un patrón de tipo 'lugar central'. Para probar esta aseveración se agrupan los datos municipales del empleo tanto en términos sectoriales como espaciales, y se utilizan indicadores de localización y de diversidad de los servicios públicos.

PALABRAS Clave | distribución espacial, economía regional, geografía económica.

ABSTRACT | Spatial patterns of the services sector have been scarcely analyzed in developing countries. In this paper, results show that the services localization follows a Central Place pattern in the case of Mexico. To prove this asseveration, employment data was aggregated in sectorial and spatial terms. Localization and diversity indicators were also used.

KEYWORDs | spatial distribution, regional economy, geographical economics.

Recibido el 13 de agosto de 2015, aprobado el 15 de julio de 2016.

E-mails: E. Pérez-Campuzano, eperez@igg.unam.mx | A. Sánchez-Zárate, alex.sanchez.zarate.2332@gmail.com |

J. Cuadrado-Roura, jr.cuadrado@uah.es 


\section{Introducción}

Los servicios son el sector más importante en términos de participación económica en el mundo. En las dos últimas décadas, entre 60\% y $68 \%$ del valor agregado mundial se generó en estas actividades, aunque tal cifra no es homogénea en todos los países. En aquellos de renta alta, el valor agregado creado en este sector llega a ser de 73\%, mientras que en países de ingreso bajo no supera el 50\%. Para México, los servicios representan 62\% del Producto Interno Bruto (Banco Mundial, 2016). No obstante, a pesar de su relevancia económica, los estudios sobre localización de servicios son escasos en países en desarrollo, y solo se han enfocado en ciertos servicios avanzados (Garza, 2006; Valdivia, 2013).

Considerando lo anterior, el presente artículo tiene como objetivo analizar la localización, especialización y diversificación del sector servicios en el caso mexicano desde una perspectiva macrorregional. Con la ayuda del Cociente de Localización y el Índice de Diversidad, se obtiene que los servicios se distribuyen espacialmente en México según los patrones especificados en la teoría del lugar central. Los servicios más especializados no solo se concentran en mayor medida en las grandes ciudades, sino que estas también presentan índices más altos de localización de los servicios. Por otro lado, las actividades que requieren menor especialización se distribuyen de manera más homogénea en el territorio. Al mismo tiempo, la diversificación económica se presenta con mayor intensidad en las zonas metropolitanas, mientras que en municipios rurales las actividades de servicios solo se especializan en servicios personales o de distribución.

El artículo se divide en cuatro apartados. El primero hace referencia a los enfoques teóricos e históricos sobre los servicios y su distribución espacial. La segunda sección describe cómo fueron agrupados los datos tanto en términos sectoriales como espaciales, así como las herramientas estadísticas utilizadas. En un tercer momento se presentan los resultados. El trabajo finaliza con unas breves consideraciones y sugerencias finales.

\section{El sector servicios: una visión geográfica}

La distribución espacial de los servicios ha ocupado un lugar importante en la geografía económica reciente (Bettencourt, Ostrom, Brown \& Roundtree, 2002; Cuadrado-Roura, 2013b; Miles et al., 1995; Moulaert \& Gallouj, 1993; Nachum, 2000; Ström, 2015; Tanaka \& Okamoto, 2008; Shearmour \& Doloreux, 2008, $2009,2014)$. Principalmente los servicios que generan alto valor agregado, como los servicios intensivos en conocimiento (KIBS, por sus siglas en inglés: knowledge intensive business services) y los servicios a las empresas, han concentrado gran parte de la atención de los investigadores (Bryson \& Daniels, 2015; Shearmur \& Doloreux, 2014; Ström, 2015). No cabe duda de que este tipo de actividades interviene de manera directa en los procesos de reestructuración/crecimiento económico de las regiones. Es por ello que se les ha vinculado con los patrones de desarrollo regional y local y sus posibilidades de futuro. Incluso se les conceptualiza como motor de 
crecimiento económico, además de cumplir con un doble rol articulador: local/ global e intersectorial.

Desde el punto de vista espacial, si bien no desaparecen factores como la (fricción de la) distancia y las economías de aglomeración, los KIBS y servicios a empresas tienen un componente claramente más cualitativo que otros y, desde luego, también suelen tener un papel más protagónico. En la actualidad, las amenidades y calidad de vida y empleo son valorados como centrales para el incremento de la productividad y la competencia entre ciudades y regiones (Shearmur \& Doloreux, 2014). En esta tarea, los citados servicios desempeñan un papel decisivo para lograr ambos objetivos.

Como se ha señalado, la mayor parte de los trabajos sobre la localización de los servicios se ha centrado en aquellos con alto valor agregado o con intensiva utilización de mano de obra más calificada. Éste ha sido también el enfoque dominante en el análisis de sus patrones espaciales (véase, entre otros, Coffey, 2000; Delgado \& García-Velasco, 2013; Hanssens, Derudder \& Witlox, 2013; Taylor, Derudder, Hoyler \& Ni, 2013). Al respecto, consideramos que el análisis de la localización de los servicios debe complementarse con la identificación del patrón de localización del resto del sector, que algunos consideran de menor importancia. ${ }^{1}$ Es decir, a diferencia de los trabajos enfocados solo en las actividades de punta, como los servicios financieros, de consultoría, inmobiliarios y tecnológicos, el presente estudio considera aquellos que son menos estudiados, como los personales o los servicios sociales. Para un resumen de las principales contribuciones en materia de distribución espacial de los servicios, véase tabla 1.

\section{Los servicios y el territorio}

Los servicios y, en particular, su localización, han figurado de manera reiterada en los estudios sobre el territorio. La teoría del lugar central (TLC), en su vertiente christalliana, ha sido la postura teórica más utilizada para explicar la configuración territorial del sector servicios (Demarco \& Matusitz, 2011; Hsu, 2012, Shearmur \& Doloreux, 2008). Este modelo pone en el centro la jerarquía urbana derivada de la diferenciación funcional de las ciudades. Sin embargo, es conocido que la formulación empírica derivada del planteamiento de Christaller no funciona para todos los casos y que las deducciones a las cuales llegó no son necesariamente las más adecuadas (Mulligan, Partridge \& Carruthers, 2012). Lo anterior no implica que se deba desechar el planteamiento teórico, sino todo lo contrario. Es de particular importancia la jerarquía urbana y su relación con la funcionalidad económica de los servicios.

Convencionalmente, la localización de los servicios se ha vinculado a factores como el tamańo del mercado (tamaño de población e ingresos), la distancia entre las empresas oferentes, la calificación de la mano de obra, la disponibilidad de medios de intercambio de mercancías, logística e infraestructura. Pero, con el cambio en

1 Según los datos de la Encuesta Nacional de Ocupación y Empleo (México, 2015), solamente el comercio en micro y pequeñas unidades económicas representa poco menos del $20 \%$ del total del empleo en el país. 
la estructura espacial de la economía, el incremento del comercio e intercambio de servicios a nivel internacional y los procesos de desindustrialización y terciarización de cada país, la distribución espacial de los servicios también se ha modificado (Cuadrado-Roura, 2013b). Particularmente los servicios avanzados tienden a la concentración espacial, debido a que requieren infraestructura especializada y un umbral mínimo de población calificada que propicie la innovación e incremento de la productividad. Por otro lado, los servicios con mayor nivel de estandarización y que requieren mano de obra menos calificada suelen desconcentrarse fácilmente. Sucede lo mismo con los servicios no comercializables (non-tradables, en inglés), los cuales se encuentran, por definición, cerca de los mercados. Así, los procesos de internacionalización de los servicios más avanzados solamente han reforzado el papel de las principales ciudades (Cuadrado-Roura, 2013a; Geppert, Gorning \& Werwatz, 2008; Searle, 1998; Tanaka \& Okamoto, 2008; Wernerheim \& Sharpe, 2005), aunque en algunos casos se pueden observar tendencias incipientes de desconcentración hacia la periferia urbana (Hermelin, 2007).

A pesar de que la TLC ha sido la más utilizada para explicar la localización de los servicios, no ha estado exenta de críticas. En la actualidad dicha postura se ha replanteado en función de los avances tecnológicos, del transporte y de los cambios en el patrón de consumo de los servicios (Cuadrado-Roura, 2013b; Mulligan et al., 2012). En este sentido, más que un apego total a la teoría descrita, el presente trabajo la recupera, debido a que explica en gran medida el comportamiento de localización del sector servicios en México desde una perspectiva de jerarquía metropolitano-urbano-rural.

Antes de pasar a estudiar el caso mexicano, es pertinente plantear algunas ideas sobre la localización de servicios. Nos referimos, por un lado, a los servicios avanzados, y por el otro, a los de menor calificación. Tomamos estos dos tipos de servicios porque constituyen un botón de muestra de la importancia cobrada por los primeros y la práctica ausencia de los segundos, desde una perspectiva espacial. 


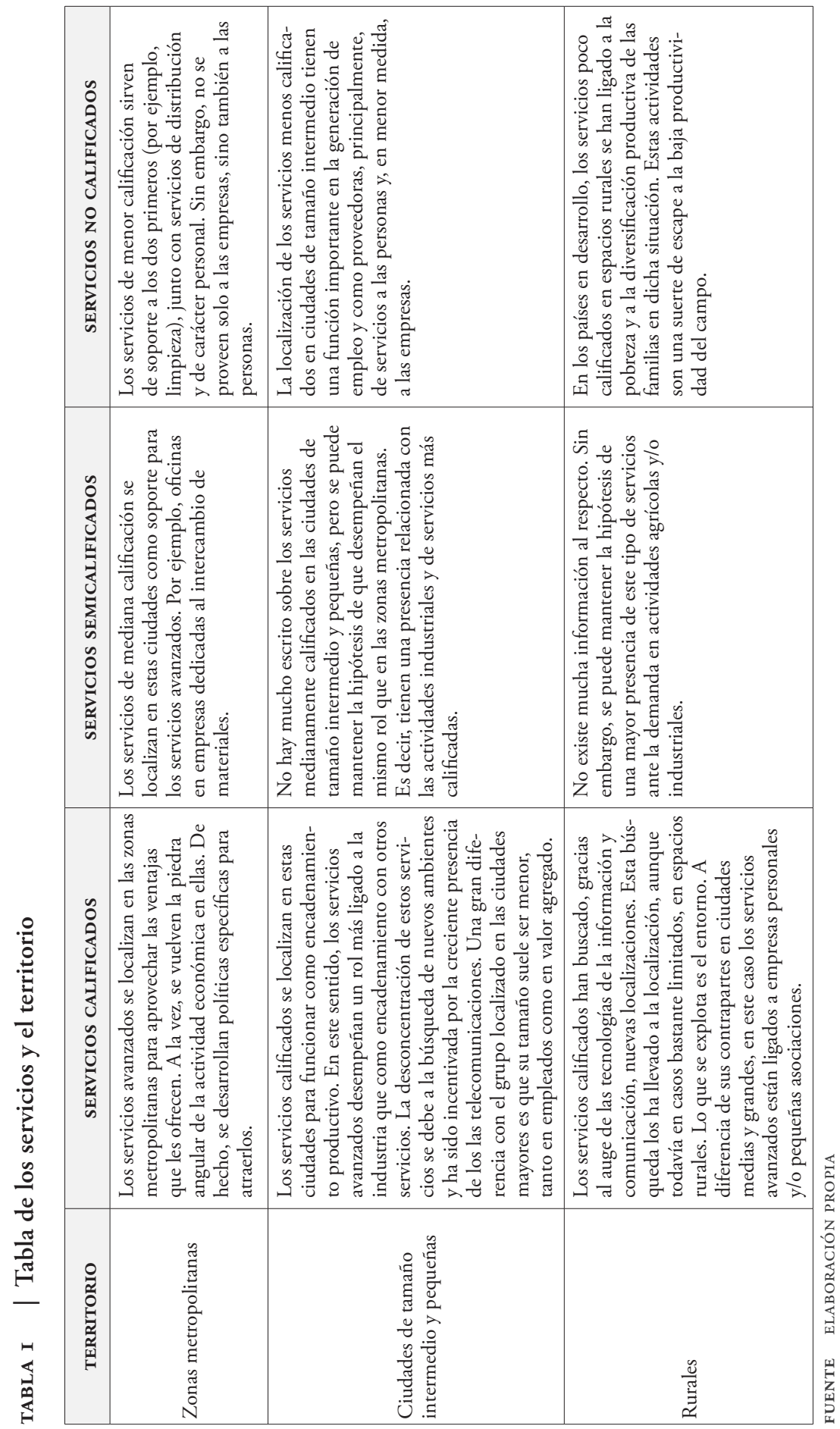




\section{Servicios intensivos en conocimiento}

Los servicios intensivos en conocimiento (KIBS) se definen como aquellas empresas, compañías u organizaciones, ya sean públicas o privadas, que prestan servicios que dependen fuertemente del conocimiento complejo o de la experiencia y cualificación específica de sus trabajadores (Shearmur \& Doloreux, 2009). Además se caracterizan por acumular, crear y difundir el conocimiento e innovación por medio de una atención personalizada y única hacia las empresas que demandan sus servicios (Bettencourt et al., 2002).

El papel de los KIBS es destacado, dado que han sido el puente entre muchas actividades que parecían desligadas entre sí. Como subrayan Taylor et al. (2013), los KIBS pueden verse como una serie de actividades que posibilitan la innovación, y con ello el desarrollo económico. Asimismo, la relevancia de los KIBs no radica únicamente en su papel de generadores y transmisores del conocimiento en términos abstractos, sino en su impacto a distintos niveles geográficos (Johansson \& Karlsson, 2009; Koch \& Stahlecker, 2006; Merino \& Rubalcaba, 2013; Wood, 2006).

La conformación espacial de estas actividades puede analizarse desde la perspectiva de la TLC y, por ende, enfatiza su explicación en el tamaño y la jerarquía de las ciudades. En esta perspectiva, los KIBS se organizan espacialmente en función de su alcance y umbral de demanda. De esta manera, se localizarán donde la demanda sea suficiente para cubrir los costos medios de producción. Dado que son servicios de alta especialización, necesitan un cierto umbral de demanda que sea también muy especializado, lo que los lleva a instalarse en las ciudades de mayor jerarquía (Parr, 2002), pues es allí donde encuentran a sus consumidores. Esto significa que el tamaño de la ciudad es un factor que explica fuertemente la localización de los servicios vinculados intensamente al conocimiento y a los negocios, aunque el gran tamaño no es la única ventaja que los lleva a instalarse en la cima del sistema territorial. En las ciudades más grandes, los KiBs tienen mayor posibilidad de interactuar con sus clientes dado que, por una parte, las tecnologías de la información y comunicación les permiten el contacto día a día; y por otra, porque allí se tiene mayor presencia de transporte aéreo, que posibilita la interrelación directa con los clientes (Shearmur \& Doloreux, 2008). Aunado a lo anterior, en las grandes ciudades se concentra la mayor parte de las personas que cuentan con capacidades para desarrollar las habilidades complejas que exige el desarrollo de estos servicios. Shearmur y Doloreux (2009, p. 85) plantean que los KIBS existen gracias a sistemas de innovación; por ejemplo, la comunicación interfirmas, las estructuras socioculturales o los ambientes de aprendizaje continuo, los cuales son más fáciles de encontrar en las ciudades.

\section{Los otros servicios: la baja calificación}

En cuanto a los servicios poco cualificados, cabe plantear tres vertientes de estudio. La primera es la presencia de este tipo de servicios en las ciudades. Los servicios poco calificados generalmente sirven de soporte para que los "avanzados" funcionen, a la vez que responden a la demanda de los ciudadanos. Por ejemplo, esa dualización del mercado del trabajo tan destacada en la literatura sobre ciudades globales (Gordon \& Kaplanis, 2014; Sassen, 2001) no hace más que plantear la necesidad que tiene 
el mercado de trabajo de contar con empleos poco remunerados y poco calificados, que sean los que sostengan a las ciudades en términos de funcionamiento, ya sea por la vía de servicios a las personas (comercio de alimentos, servicios personales) o a las empresas (limpieza, reparaciones, seguridad y plomería, por mencionar solo algunos). En el caso de los estudios urbanos, la existencia de empleos de poca calificación/productividad del sector servicios no es un tema nuevo, pero sí lo son las expresiones que esto ha tomado en los últimos años. Las ciudades pasan por un proceso de reestructuración económica que ha dado lugar a una mayor desigualdad, la cual se ha expresado -entre otras vertientes- en el gran incremento de categorías de empleo en actividades con poco valor agregado y/o baja productividad.

La segunda línea de estudio remite a los servicios no comercializables y que tienen un patrón más desconcentrado, puesto que requieren de la concurrencia de proveedores y consumidores que suelen estar distribuidos según lo requerimientos del mercado. Este tipo de servicios se caracteriza por la menor calificación laboral promedio de sus empleados, en comparación con los anteriores (Gervais \& Jansen, 2013), y por una distribución espacial más homogénea.

Por último, se encuentran los servicios poco calificados ubicados en las zonas rurales. Tanto en países en desarrollo como en los desarrollados, los servicios se han convertido en uno de los principales tipos de actividad que crean empleo en estas áreas. En Estados Unidos, el comercio al menudeo es el principal empleador en las áreas rurales (Vias, 2004) y en Dinamarca el empleo en este tipo de servicios es de aproximadamente $60 \%$ en las comunidades no mayores de 5.000 habitantes (Illeris, 2005). Esta misma proporción se encuentra en numerosos países desarrollados y/o en vías de desarrollo. Lo que comparten dichas cifras es una tendencia hacia la dispersión de empleos menos calificados en los ámbitos más rurales (Illeris, 2005). En las economías desarrolladas, la desconcentración de los servicios ha venido de la mano de la migración hacia estos espacios, del envejecimiento poblacional y de la relocalización de algunas industrias (Miller \& Bluestone, 1988). En los países en vías de desarrollo, el crecimiento de los servicios en ámbitos rurales ha sido resultado de la búsqueda de alternativas a la situación de pobreza en la que vive gran parte de la población y, por supuesto, motivado también por la necesidad de atender las demandas de su población.

\section{Los servicios en México}

$\mathrm{Al}$ igual que sucede y ha sucedido en otros países, en México el tránsito hacia una sociedad de servicios es evidente (Garza, 2006). En la década de los ochenta, el crecimiento de los servicios había sido conceptualizado como 'terciarización', e incluso llegándose a colocar prefijos como "híper" para denotar lo que se calificaba como excesiva participación del sector servicios en la economía del país. Con posterioridad a la publicación de La cuestión urbana (Castells, 1977) y a la implantación de la noción de hiperterciarización, la mayor parte de los estudios se concentró en la poca capacidad de las economías no desarrolladas para absorber la sobreoferta de mano de obra. En este caso, la terciarización era vista como un proceso inacabado de industrialización, ligado a una alta primacía urbana. Posteriormente, la discusión sobre la terciarización se unió a la informalización de la mano de obra. 
La terciarización implicaba dos aspectos estrechamente vinculados. Por un lado, un proceso de ajuste económico, donde se expandía el empleo en un sector no productivo o, al menos, con una baja productividad (Baumol, 1967); por el otro, estaban las políticas de ajuste estructural, que implicaban la expulsión de mano de obra de la industria y la disminución de las prestaciones sociales. Influidos por los textos de Portes (1995), los estudios ligaban la expansión del empleo informal al sector terciario. Por ejemplo, son característicos los textos sobre la informalidad en el caso de los vendedores de calle (llamados 'ambulantes').

Hacia la década de los noventa, pueden encontrarse las primeros cambios en la visión del papel de los servicios en el desarrollo económico de las ciudades (Parnreiter, 2002; Graizbord et. al., 2003). Se planteó ir más allá de una visión de los servicios como dependientes de la industria, para insertarlos en el contexto de la globalización y los procesos de crecimiento regional (Garza, 2006), además de adentrarse en la multiplicidad de aspectos vinculados a la relación entre espacio y servicios (Garza, 2006, 2009; Graizbord, Rowland \& Aguilar, 2003; Parnreirter, 2002; Valdivia, 2013). Los servicios dejaron de ser una suerte de lastre económico, para convertirse en la punta de lanza de las nuevas economías.

Sin embargo, la gran mayoría de los estudios se ha centrado en ciudades/entidades específicas (Garza, 2006, 2009; Valdivia, 2013) y han dejado de lado una visión general del sector servicios en el país desde una vertiente espacial. Un grupo de trabajos se ha concentrado en el papel activo de los servicios en la articulación entre las economías locales y los procesos de ajuste global (denominada, en términos genéricos, como globalización) (Graizbord et al., 2003; Parneirter, 2002). En otros casos, la forma de analizar los servicios ha sido desde una perspectiva intraurbana y considerando cómo ellos configuran cierto tipo de estructuras espaciales (Garza, 2006, 2009; Valdivia, 2013). Por ejemplo, Valdivia (2013) plantea que los servicios creativos contribuyen al crecimiento económico de las zonas metropolitanas analizadas y que su distribución espacial se encuentra claramente localizada en los centros económicos de las mismas.

Dados estos antecedentes teóricos y empíricos, cobra sentido el estudio del sector servicios avanzados e inferiores desde una perspectiva geográfica que analice a todo el país.

\section{Base de datos y metodología para el análisis espacial de los servicios en México}

Los datos de base utilizados a efectos de nuestro análisis tienen una desagregación municipal. Los 2.456 municipios mexicanos se han agrupado en cinco categorías, de acuerdo con su carácter metropolitano o su talla demográfica:

1. Zonas Metropolitanas. Esta categoría se construyó con la clasificación mexicana de Zonas Metropolitanas elaborada por la Secretaría de Desarrollo Social (sEDesol), el Consejo Nacional de Población (CONAPo) y el Instituto Nacional de Geografía y Estadística (INEGI), e incluye 59 zonas metropolitanas, compuestas 
por 367 unidades político-administrativas. Como caso particular, las zonas metropolitanas se han subdividido en:

1.1 Zona Metropolitana de la Ciudad de México (zMCM). Dado su gran peso, se ha decidido construir una categoría especial para ella; además, permite observar tendencias de desconcentración en el periodo de análisis.

1.2 Las 58 zonas metropolitanas restantes.

2. Los municipios urbanos se definieron como aquellos con al menos una localidad de más 15.000 habitantes y que no formaban parte de una zona metropolitana.

3. Los municipios rurales son todos aquellos sin localidades superiores a 14.999 habitantes y que no se encuentran en alguna zona metropolitana.

De esta manera, el territorio mexicano se ha divido en 59 zonas metropolitanas, 304 municipios urbanos y 1785 municipios rurales.

Para medir la actividad en servicios, se utiliza como variable el personal ocupado. Dicha información se obtuvo de los censos económicos de 1999, 2004 y 2009 publicados por el INEgi, el cual utiliza el Sistema de Clasificación Industrial de América del Norte (sCIAN). Los servicios se han organizado en cinco grupos siguiendo el trabajo de Miller y Bluestone (1989) (tabla 2): servicios tradicionales a la producción, servicios intensivos en conocimiento, servicios de distribución, servicios sociales y servicios personales.

\section{TABLA 2 Clasificación de servicios según grupos}

\begin{tabular}{|l|l|l|}
\hline \multirow{2}{*}{$\begin{array}{l}\text { Servicios a la } \\
\text { producción }\end{array}$} & $\begin{array}{l}\text { Servicios tradicionales } \\
\text { a la producción (STP) }\end{array}$ & $\begin{array}{l}\text { Son todos aquellos que se han considerado como servicios a las } \\
\text { empresas, aunque generalmente tienen un rol menos importante } \\
\text { en la generación y utilización de información. }\end{array}$ \\
\cline { 2 - 3 } Servicios de distribución & $\begin{array}{l}\text { Servicios intensivos en } \\
\text { conocimiento (KIBS) }\end{array}$ & $\begin{array}{l}\text { Servicios con intensidad importante en la generación/utilización } \\
\text { de información, y con exigencias más elevadas en términos de } \\
\text { formación de sus empleados. }\end{array}$ \\
\hline Servicios sociales & $\begin{array}{l}\text { Servicios que tienen como finalidad el servir como } \\
\text { infraestructura o medios para la movilidad de productos y/o } \\
\text { personas. }\end{array}$ \\
\hline Servicios personales & $\begin{array}{l}\text { Servicios que tienen como finalidad la reproducción social vía la } \\
\text { mejora de las condiciones de vida de la población. Esta categoría } \\
\text { está marcada por los servicios educativos y de salud, sean } \\
\text { provistos por el gobierno o por empresas privadas. }\end{array}$ \\
\hline & $\begin{array}{l}\text { Se encuentran todas aquellas actividades cuya finalidad es } \\
\text { proveer un servicio/bien a las familias de manera tal que no } \\
\text { se incluyen en todos aquellos considerados como básicos } \\
\text { (educación y salud). }\end{array}$ \\
\hline
\end{tabular}

FUENTE ELABORACIÓN PROPIA CON BASE EN MILLER Y BLUESTONE (I989)

En lo que respecta a las herramientas estadísticas para identificar los patrones espaciales, en este trabajo se utilizan dos índices para medir concentración y diversidad de los servicios en México: el cociente de localización (QL) y el índice de Shannon (IS). 
El QL ha sido utilizado reiteradamente en los estudios de concentración, y se define como:

$$
Q L_{i j}=\frac{x_{i j}}{\sum_{i=1}^{R} x_{i j}} / \frac{\sum_{j=1}^{M} x_{i j}}{\sum_{i=1}^{R} \sum_{j=1}^{M} x_{i j}}
$$

donde:

$\mathrm{Xij}_{\mathrm{ij}}=$ Empleo en la unidad espacial $\mathrm{j}$ en el sector $\mathrm{i}$

El QL se utiliza como medida de especialización de la actividad económica en el territorio. De esta manera, si la proporción del sector i en la unidad espacial j es superior a la proporción del total nacional del sector i en el total de empleo del país, el QL será superior; por lo tanto, dicha actividad se encontrará especializada de manera relativa.

El Is, que se toma como referencia para el análisis de la diversidad económica, es un índice ampliamente utilizado en biología para conocer la riqueza de especies, pero que también ha sido empleado recientemente para determinar la distribución de las actividades económicas o de la población (Pérez, 2010). De manera formal:

$$
I S=-\sum_{i=1}^{S} p_{i j} \log \left(p_{i j}\right)
$$

donde:

$\mathrm{p}_{\mathrm{i}}=$ proporción de empleados en el sector i respecto al total de empleo en la unidad $\mathrm{j}\left(\mathrm{n}_{\mathrm{ij}} / \mathrm{N}_{\mathrm{j}}\right)$

$S=$ total de sectores

Con ambos métodos pretendemos analizar la especialización y diversificación de los servicios en el territorio mexicano. La combinación de las técnicas con la representación cartográfica permitirá visualizar de manera espacial el comportamiento del sector terciario.

\section{Resultados y discusión}

La estructura del empleo en la economía mexicana es evidentemente de carácter terciario; así, en 2009, el 69\% del empleo generado provenía de este sector (Ortiz, 2006). La figura 1 muestra que a lo largo de diez ańos, la participación de los diversos grupos de servicios se ha mantenido relativamente estable, siendo el grupo de servicios de distribución el que concentra prácticamente la mitad del empleo terciario. Al mismo tiempo, México comparte con gran parte de los países en vías de 
desarrollo la poca calificación de su sector servicios (Einchengreen \& Gupta, 2013; World Bank, 2008). Este aspecto ha continuado a lo largo del periodo de análisis. En términos generales, se aprecia una relación inversa entre el nivel de empleo generado y el grado de especialización de los servicios. De esta manera, las actividades más rutinarias, como los servicios de distribución o personales, son mayores generadores de empleo, mientras que los servicios más especializados tienen menor participación en el empleo. Este hecho se explica por ser el sector terciario el grupo con mayor número de ramas de actividad y porque en él se encuentra el comercio, que ocupa la mayor cantidad de población en México.

La figura 1 también muestra que las actividades que menos empleo generan son los STP, los servicios sociales y los KIBs. Una conclusión importante en este sentido es que si bien es cierto que los servicios avanzados tienen tendencia a una mayor participación en países en desarrollo, en México siguen un patrón con empleos de baja productividad, pues de la mano de la poca participación de las actividades avanzadas de servicios viene la escasa calificación de la mano de obra en estas actividades. Por ejemplo, según los datos de la Encuesta Nacional de Ocupación y Empleo (enoe) 2015, en las categorías de Servicios de Distribución y Servicios Personales el porcentaje de población calificada alcanzó el 5\% del total; mientras que en KIBS esta cifra es de $21 \%$, lo que influye directamente en la productividad laboral. Es por ello que se hace necesaria una intervención importante para desarrollarlos en el mediano y largo plazo.

FIGURA I | Participación porcentual según grupos de servicios a nivel nacional, 1999, 2004 y 2009

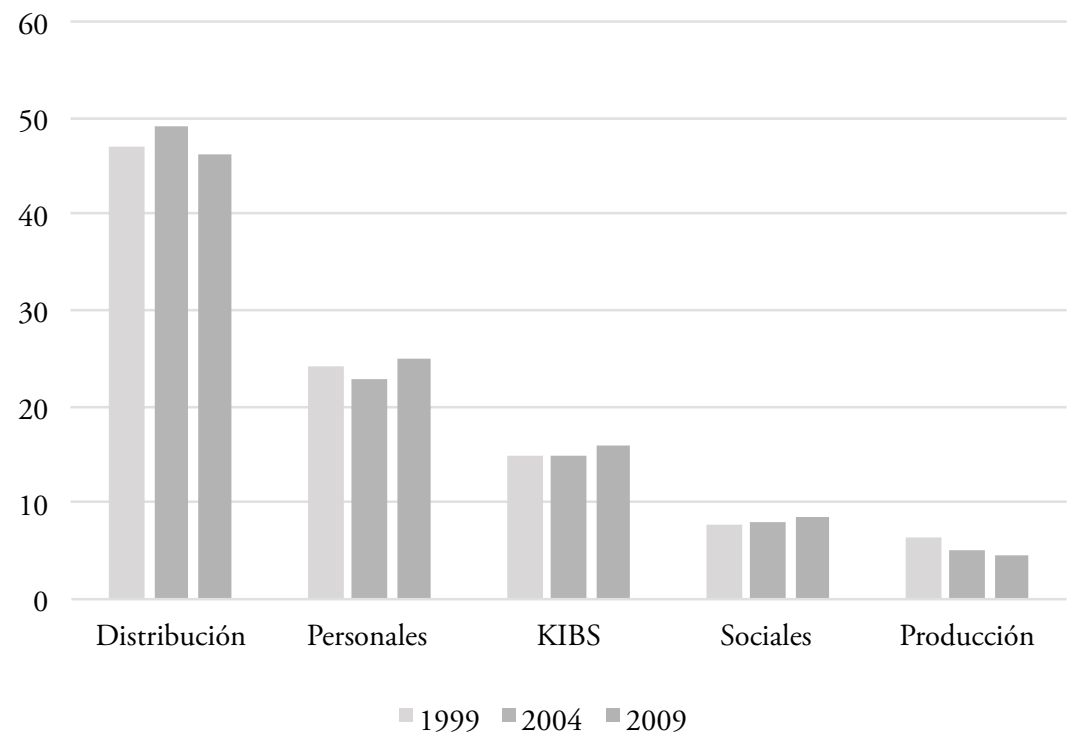

FUENTE ELABORACIÓN PROPIA CON DATOS DE CENSOS ECONÓMICOS I 999, 2004 Y 2009 
La distribución espacial de servicios presenta un patrón de alta concentración en las ciudades de mayor tamaño, particularmente en la zMcM. Ya sea que esta formación se deba a la búsqueda de economías de escala y aglomeración u obedezca a la propia distribución espacial de la población, los servicios se encuentran concentrados en las 59 zonas metropolitanas. Del total de la población ocupada en el sector servicios, tres cuartas partes se localizan en alguna de las urbes. Sin embargo, este patrón de concentración es más acentuado en los servicios avanzados y hay una cierta desconcentración en los de menor productividad, como se mostrará adelante.

La distribución espacial de los servicios avanzados presenta un esquema tradicional, es decir, de alta concentración en ciudades de mayor tamańo, lo que implica que se ubican en pocas ciudades del país, mientras que aquellos con menor nivel de productividad tienen una distribución más homogénea en el territorio. En este tenor, las zonas metropolitanas de mayor tamańo concentran la mayor parte del empleo en KIBS y también de los STP. Esta concentración de servicios altamente calificados y con una participación importante en el Valor Agregado contribuye, sin duda, a que se incrementen las disparidades interregionales en México.

TABLA 3 Participación porcentual de los servicios según grupos de agregación y distribución espacial, 1999, 2004 y 2009

\begin{tabular}{|c|c|c|c|c|c|c|}
\hline & \begin{tabular}{|c|} 
SERVICIOS \\
TRADICIONALES \\
A LA \\
PRODUCCIÓN
\end{tabular} & $\begin{array}{c}\text { SERVICIOS } \\
\text { INTENSIVOS EN } \\
\text { CONOCIMIENTO } \\
\text { (KIBS) }\end{array}$ & $\begin{array}{c}\text { SERVICIOS } \\
\text { DE } \\
\text { DISTRIBUCIÓN }\end{array}$ & $\begin{array}{l}\text { SERVICIOS } \\
\text { SOCIALES }\end{array}$ & $\begin{array}{c}\text { SERVICIOS } \\
\text { PERSONALES }\end{array}$ & $\begin{array}{c}\text { TOTAL } \\
\text { NACIONAL }\end{array}$ \\
\hline \multicolumn{7}{|l|}{ I999 } \\
\hline ZMCM & 29,5 & 38,8 & 25,4 & 28,8 & 28,6 & 28,7 \\
\hline \begin{tabular}{|l|} 
Zonas \\
metropolitanas
\end{tabular} & 48,1 & 44,3 & 45,3 & 50,1 & 44,5 & 45,5 \\
\hline \begin{tabular}{|l|} 
Municipios \\
urbanos
\end{tabular} & 18,4 & 13,8 & 22,2 & 17,5 & 21,2 & 20,1 \\
\hline \begin{tabular}{|l|}
$\begin{array}{l}\text { Municipios } \\
\text { rurales }\end{array}$ \\
\end{tabular} & 4,0 & 3,1 & 7,2 & 3,5 & 5,7 & 5,7 \\
\hline Total & 100,0 & 100,0 & 100,0 & 100,0 & 100,0 & 100,0 \\
\hline \multicolumn{7}{|l|}{2004} \\
\hline ZMCM & 26,6 & 39,7 & 24,8 & 28,5 & 27,6 & 28,0 \\
\hline \begin{tabular}{|l|} 
Zonas \\
metropolitanas \\
\end{tabular} & 50,5 & 44,1 & 45,6 & 50,7 & 45,1 & 45,9 \\
\hline \begin{tabular}{|l|} 
Municipios \\
urbanos
\end{tabular} & 19,4 & 13,4 & 22,3 & 17,7 & 21,5 & 20,3 \\
\hline $\begin{array}{l}\text { Municipios } \\
\text { rurales }\end{array}$ & 3,5 & 2,8 & 7,4 & 3,1 & 5,8 & 5,8 \\
\hline Total & 100,0 & 100,0 & 100,0 & 100,0 & 100,0 & 100,0 \\
\hline \multicolumn{7}{|l|}{2009} \\
\hline ZMCM & 26,3 & 40,3 & 23,3 & 24,6 & 25,9 & 26,9 \\
\hline \begin{tabular}{|l} 
Zonas \\
metropolitanas
\end{tabular} & 51,5 & 43,8 & 45,4 & 52,8 & 44,3 & 45,8 \\
\hline $\begin{array}{l}\text { Municipios } \\
\text { urbanos }\end{array}$ & 19,3 & 13,5 & 22,7 & 18,9 & 23,1 & 20,8 \\
\hline \begin{tabular}{|l|}
$\begin{array}{l}\text { Municipios } \\
\text { rurales }\end{array}$ \\
\end{tabular} & 2,9 & 2,4 & 8,6 & 3,7 & 6,7 & 6,4 \\
\hline Total & 100,0 & 100,0 & 100,0 & 100,0 & 100,0 & 100,0 \\
\hline
\end{tabular}

FUENTE ELABORACIÓN PROPIA A PARTIR DE LOS CENSOS ECONÓMICOS I999, 2004 Y 2009 
Nuestro análisis lleva a comprobar que el empleo en los STP se genera, principalmente, en las zonas metropolitanas y en los municipios urbanos. Con excepción de la ZMCM, las metrópolis mexicanas captaron en 1999 un 48\% del empleo de este grupo, llegando a representar, una década después, un $51 \%$ del mismo. Esto se explica por el incremento de la demanda de estos servicios, así como por el proceso de desconcentración de los stp hacia ciudades de menor rango. ${ }^{2}$ En este sentido, estas actividades han buscado el vínculo con actividades industriales y la desconcentración que estas últimas han experimentado en las últimas décadas (Aguilar, 1999).

Por otro lado, los KIBs presentan alta concentración en la ZMCM (tabla 3), con cifras que van aumento. Así, si en 1999 representaban el 38,8\% de los servicios, en 2009 alcanzaban el 39,7\%, y ello con altas tasas de crecimiento (alrededor de 6\% anual entre ambos ańos). En otras palabras, no existe una relación entre tamaño poblacional y participación en esta categoría de servicios de la ZMCM. Ahora bien, si se toma la totalidad de las zonas metropolitanas, ellas agrupan $83 \%$ del total de esta categoría. Por su parte, los municipios urbanos tienen una participación constante de KIBS de alrededor de $13 \%$.

En contraposición al caso anterior se encuentran los servicios de distribución, que se localizan de manera menos concentrada. Las zonas metropolitanas y los municipios urbanos representan, en conjunto, un 92\% del empleo en actividades de distribución en 671 municipios. De este porcentaje, un $45 \%$ corresponde a las zonas metropolitanas, con excepción de la ZMCM; a su vez, en dichos municipios habita un 80\% de la población total del país. Las actividades de distribución se vinculan directamente al tamańo demográfico, aunado a que es la actividad terciaria que genera mayor empleo.

Los servicios sociales y de carácter personal presentan un patrón similar a los de distribución. La ZMCM mantiene su posición predominante, con una ligera pérdida de peso relativo durante la década que estamos considerando (aproximadamente de $4 \%$ entre 1999 y 2009), y las zonas metropolitanas y los municipios urbanos y rurales incrementan marginalmente su peso. Lo que es importante notar es que ambas categorías son las que menos cambios presentan a lo largo de los diez años del estudio; en otras palabras, el crecimiento ha sido más o menos homogéneo para las cuatro categorías (tabla 4).

TABLA 4 Tasas de crecimiento promedio anual según grupos de agregación y distribución espacial, 1999-2009

\begin{tabular}{|l|c|c|c|c|c|c|}
\cline { 2 - 7 } \multicolumn{1}{c|}{} & STP & KIBS & $\begin{array}{c}\text { SERVICIOS DE } \\
\text { DISTRIBUCIÓN }\end{array}$ & $\begin{array}{c}\text { SERVICIOS } \\
\text { SOCIALES }\end{array}$ & $\begin{array}{c}\text { SERVICIOS } \\
\text { PERSONALES }\end{array}$ & $\begin{array}{c}\text { TOTAL } \\
\text { SERVICIOS }\end{array}$ \\
\hline ZMCM & 0,42 & 6,48 & 3,95 & 4,83 & 4,68 & 4,55 \\
\hline Zonas metropolitanas & 2,28 & 5,98 & 4,89 & 7,07 & 5,68 & 5,29 \\
\hline Municipios urbanos & 2,10 & 5,86 & 5,12 & 7,33 & 6,62 & 5,60 \\
\hline Municipios rurales & $-1,77$ & 3,42 & 6,83 & 6,96 & 7,51 & 6,49 \\
\hline
\end{tabular}

FUENTE ELABORACIÓN PROPIA A PARTIR DE LOS CENSOS ECONÓMICOS I 999 Y 2009 
Si se analizan los cambios en términos de tasas de crecimiento, se observa el mismo patrón (tabla 4). Mientras que las actividades de mayor generación de empleo (sTP y KIBS) presentan una tendencia al crecimiento en las ciudades de mayor tamańo, los servicios personales, sociales y de distribución son los más dinámicos en los municipios rurales. El incremento de estas categorías se puede explicar por la situación de mayor pobreza que existe en los ámbitos rurales -la dinámica económica de las familias en pobreza se liga a la diversificación productiva, generalmente en actividades que requieren poca inversión-, así como por la necesidad de los gobiernos de llevar determinados servicios básicos (educación y salud) a esta población.

\section{Especialización y diversificación del sector servicios en México}

Hasta aquí hemos hecho referencia, de forma general, a las participaciones del empleo terciario en el territorio mexicano, tomando en cuenta las principales actividades que componen los servicios. Sin embargo, esto no permite desentrańar el comportamiento espacial de concentración o desconcentración de este sector en México, por lo que a continuación se presentan los resultados de los cocientes de localización (QL) y del índice de Shannon (IS).

La concentración económica ha sido utilizada para conocer cómo determinadas regiones/ciudades se especializan en ciertas actividades. En el caso de una visión que privilegia la jerarquía, se espera que las actividades de servicios avanzados se encuentren preferentemente localizadas en los grandes centros urbanos, mientras que los servicios menos avanzados sean más homogéneos en el territorio (figura 2a-2c y tabla 5).

FIGURA 2 | Distribución espacial de la especialización en KIBS, 1999-2009

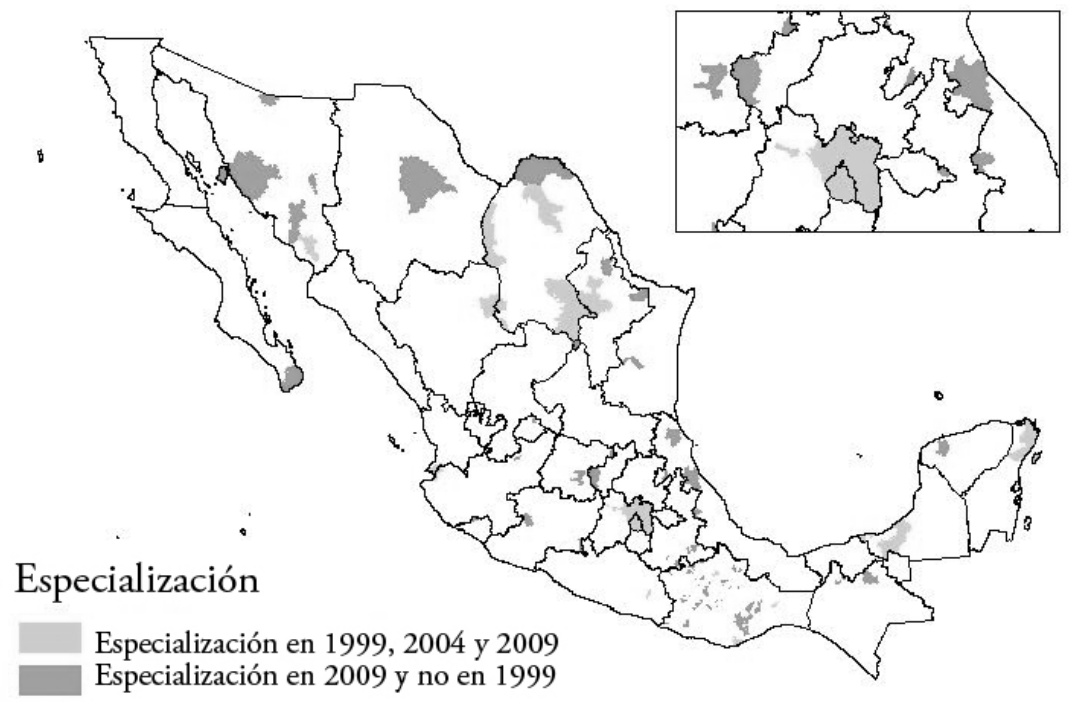

FUENTE ELABORACIÓN PROPIA CON DATOS DE CENSOS ECONÓMICOS I999, 2004 Y 2009 


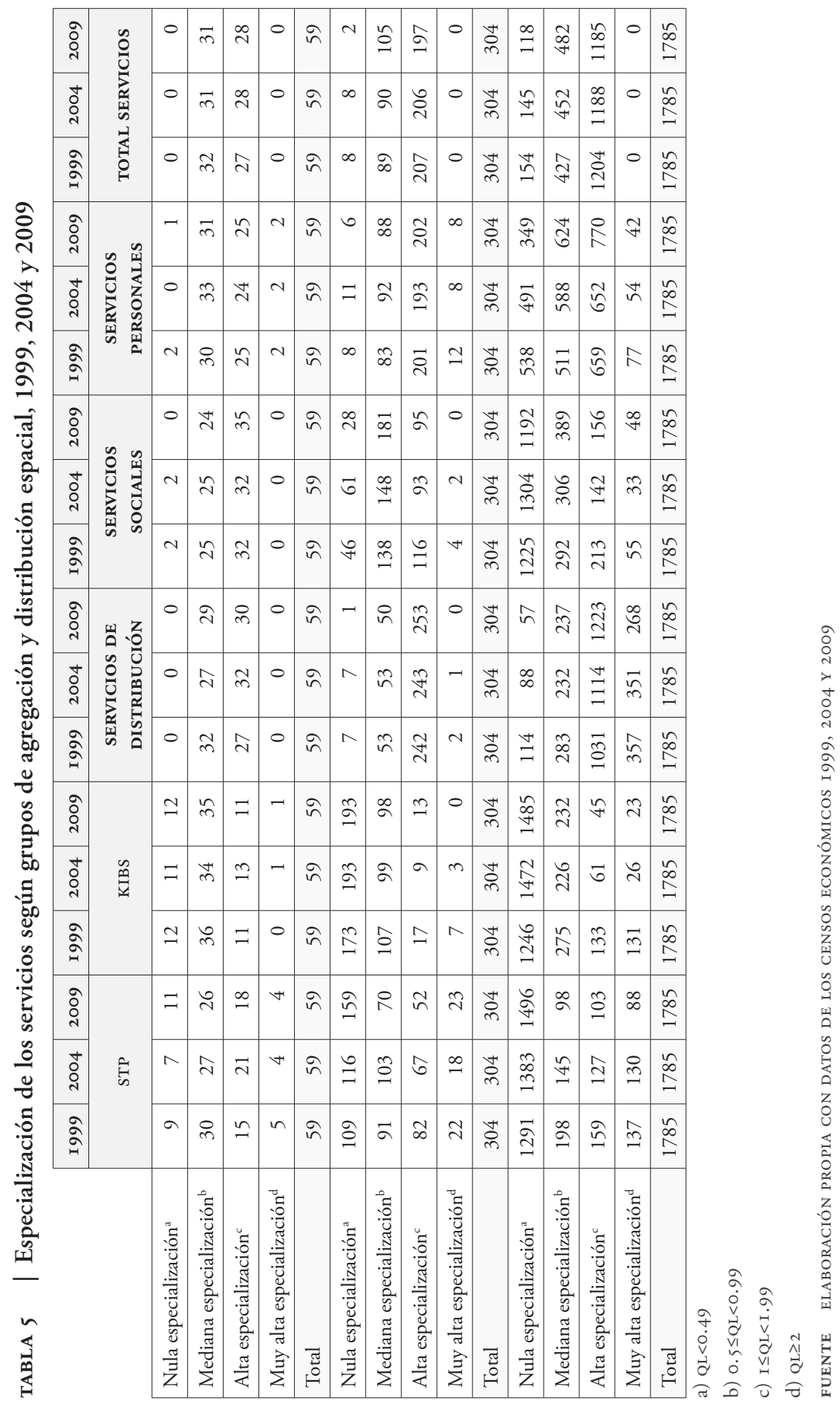


FIGURA 3 Especialización en STP (2a), KIBS (2b), servicios personales (2c) y diversificación económica $(2 \mathrm{~d}), 2009$

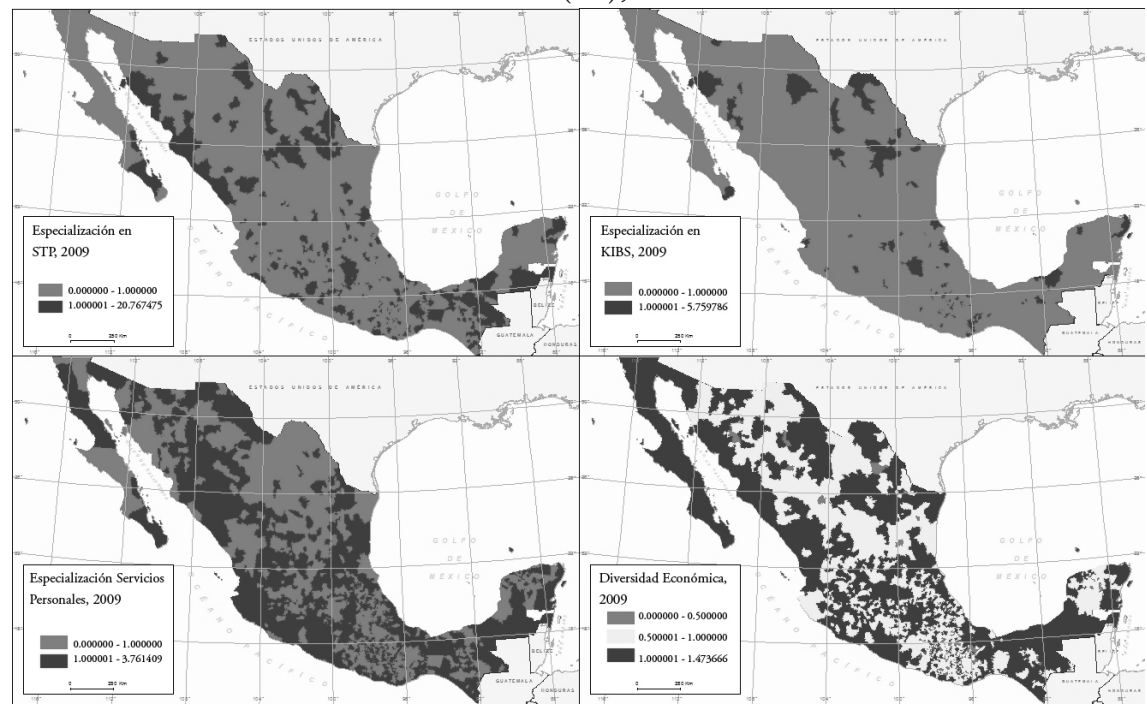

FUENTE ELABORACIÓN PROPIA CON DATOS DE LOS CENSOS ECONÓMICOS I999, 2004 Y 2009

El análisis empírico permite afirmar que, con carácter general, en México los servicios se distribuyen como lo establece la TLC, aunque con algunas matizaciones importantes. Los servicios más especializados son los KIBs. La figura 3 muestra que muy pocas ciudades se especializan en estas actividades y su distribución se explica por tres elementos relevantes: la jerarquía y talla demográfica de las ciudades; el carácter internacional de las ciudades; y el patrón espacial de la industria. Las zonas metropolitanas de la Ciudad de México y Monterrey se categorizan en el primero de dichos elementos, aunque también juegan un importante papel los otros dos. Ambas ciudades son las más grandes del país y cumplen, al mismo tiempo, con la característica de tener vínculos muy estrechos con la economía internacional. En el caso de Cancún, en el sureste mexicano, la especialización en KIBs se debe, esencialmente, a su carácter de centro turístico internacional. Finalmente en otras zonas metropolitanas, como Saltillo, La Laguna, y en ciudades como Navojoa, todas ellas en el norte del país, su especialización en estos servicios intensivos en conocimiento se relaciona -sobre todo- con el desarrollo que allí existe de la industria o de la agricultura.

La figura 3 complementa el análisis de la especialización de los KIBs en este periodo. La ganancia en especialización se debe a la transformación espacial de la industria de algunas ciudades. Particularmente es en el Golfo Mexicano y el norte del país donde el mayor número de unidades adquieren alta especialización en 2009. Como se muestra, la alta y muy alta especialización de los KIBS es constante en las zonas metropolitanas y municipios urbanos arriba mencionados. Adicionalmente, se observa que este tipo de servicios altamente especializados empieza a cobrar importancia en municipios o metrópolis que no se ubican en la cima del sistema 
urbano mexicano, como en el caso de los municipios de los estados de Oaxaca, Chiapas, Sonora, Chihuahua o Veracruz. En este sentido, este tipo de servicios se da en función de otros elementos, más allá del tamaño poblacional.

La dinámica de coaglomeración es clara en las grandes ciudades y no así en las ciudades pequeñas y ámbitos rurales. Las zonas metropolitanas tienen una mayor probabilidad de especializarse en más de una actividad, incluyendo muy altos índices de especialización en servicios a las empresas y servicios intensivos en conocimiento (figura 2a-2b). Esta diversificación que ocurre en grandes ciudades es examinada por Duranton y Puga (2000), que la explican en tanto vinculada a su mayor capacidad innovativa y de competencia económica. Al mismo tiempo, también es congruente con los postulados de la teoría del lugar central (TLC), ya que las entidades de mayor jerarquía tendrán presencia no solo de las actividades de mayor especialización, sino también de las de menor importancia. Por su parte, los municipios rurales se especializan en actividades con poco valor agregado y un máximo de dos actividades (servicios personales, principalmente) (figura 2a-2c).

Por otro lado, los servicios con poco valor ańadido y/o no comerciables tienen una distribución espacial más homogénea en México. En términos porcentuales, entre $77 \%$ y $83 \%$ de los municipios rurales están especializados en servicios de distribución, mientras que entre $40 \%$ y $45 \%$ de los municipios de esta misma categoría se especializan en servicios personales. Cabe incluso subrayar, con carácter más general, que $66 \%$ de estos se especializa en servicios debido a que gran parte de sus actividades productivas recaen en el sector terciario.

En contraposición, si bien las grandes ciudades son las únicas que se especializan en los KIBS y STP, también tienen en ellas una fuerte presencia el resto de las actividades de servicios. Más de la mitad de las zonas metropolitanas tiene un nivel de especialización alto y muy alto en este sector en su conjunto. Estos resultados son congruentes con el estudio de Angoa, Pérez-Mendoza y Polèse. (2009), en el que los servicios se analizan tanto desde la perspectiva regional/urbana como desde el punto de vista sectorial.

Si se analizan los cambios desde una perspectiva diacrónica, se aprecia que no son significativos. Esto implica que las ventajas creadas con anterioridad se han visto reforzadas. Por ejemplo, los servicios a la producción refuerzan su patrón concentrador en las grandes zonas metropolitanas y en algunos municipios urbanos, mientras que los municipios rurales siguen mostrando el patrón -ya subrayado anteriormente- de empleos poco productivos. De manera amplia, se ha visto que las zonas metropolitanas y los municipios urbanos tienen la capacidad de especializarse en más de un grupo de servicios, aunque el nivel de diversificación no sea el mismo a lo largo de todo el territorio.

\section{Diversidad económica}

El último indicador que hemos utilizado en nuestro análisis es el de diversidad económica. Mucho se ha insistido en la importancia que tiene la concentración/ especialización en la formación de estructuras espaciales; sin embargo, poco se ha planteado sobre la diversidad económica. En este caso, en un primer acercamiento al tema, planteamos que la diversidad económica tiene relación con la especialización. 
La diversidad económica ha mostrado ser un elemento detonante de la innovación en ciudades (Bettencourt et al., 2002), pues posibilita los intercambios no únicamente personales, sino sobre todo entre actividades complementarias, aunque no necesariamente dentro del mismo sector.

Como sucede con la especialización, la diversidad económica se encuentra claramente marcada por una diferenciación espacial jerárquica. Mientras que las zonas metropolitanas tienden a diversificarse, los municipios rurales dependen de un número menor de actividades (figura $2 \mathrm{~d}$ y tabla 6 ). Como ha sido analizado en otros estudios (Bettencourt, et al. 2002), la diversificación es uno de los factores que detonan la innovación y el desarrollo económico de las ciudades. Es por ello tan importante que exista este factor. En este sentido, se puede plantear que la diversificación productiva se convierte en el puente entre crecimiento económico e innovación. Por otro lado, la diversificación productiva en espacios rurales se relaciona con la pobreza y la búsqueda de salidas a ella. ${ }^{3}$

La tabla 6 y la figura $2 \mathrm{~d}$ ofrecen perspectivas complementarias al estudio de la diversificación económica de los servicios. Por un lado, todas las zonas metropolitanas se encuentras diversificadas o altamente diversificadas. Asimismo, la diversificación de las zonas metropolitanas se debe a la relación interurbana que experimentan a escala regional. En este punto, la figura $2 \mathrm{~d}$ permite visualizar la alta diversificación de algunas zonas, que obedece a factores regionales dentro del país. Bajo esta premisa, se identifican cinco conglomerados de diversificación económica: Norte-Pacífico, Norte-Golfo, Centro-Bajío, Centro-Pacífico, Sur-Este y Caribe. En cada uno de ellos, la diversificación se relaciona con diferentes factores. Así, en la región Norte los servicios se vinculan más a actividades industriales; en la CentroBajío y Centro-Pacífico, a industria, servicios y turismo; y la diversificación del Caribe, a actividades turísticas.

Por otro lado, si se presentan los resultados de manera diacrónica, se hace evidente que los mayores incrementos en la diversificación productiva han tenido lugar en los municipios urbanos. En otras palabras, la diversidad económica ya era un factor importante en las economías metropolitanas, pero poco a poco los municipios urbanos parecen acrecentar cada vez más esta característica, lo cual teóricamente debiera influir en la localización de los servicios con mayor valor agregado. También habrá que llamar la atención sobre el hecho de que tres cuartas partes de los municipios rurales figuran en la categoría de medianamente especializados, lo que reforzaría la hipótesis del incremento de la presencia de actividades no agrícolas en este tipo de espacios, aunque -como ya se planteó- no necesariamente son las que tienen un mayor valor agregado. diversificados son generalmente los que presentan los índices de marginación más altos. En este sentido, el tema nos puede llevar a la discusión de la importancia de la diversificación económica y su relación con las políticas antipobreza en países como México. 
TABla 6 | Diversidad económica según distribución espacial, 1999, 2004 y 2009

\begin{tabular}{|c|c|c|c|}
\hline & I999 & 2004 & 2009 \\
\hline \multicolumn{4}{|l|}{ Zonas metropolitanas } \\
\hline Muy poco diversificadas ${ }^{a}$ & 0 & 0 & 0 \\
\hline Poco diversificadas $^{\mathrm{b}}$ & 0 & 0 & 0 \\
\hline Medianamente diversificadas ${ }^{c}$ & 6 & 5 & 4 \\
\hline Diversificadas $^{\mathrm{d}}$ & 53 & 54 & 51 \\
\hline Altamente diversificadas ${ }^{\mathrm{e}}$ & 0 & 0 & 4 \\
\hline \multicolumn{4}{|l|}{ Municipios urbanos } \\
\hline Muy poco diversificados ${ }^{a}$ & 1 & 1 & 0 \\
\hline Poco diversificados $^{\mathrm{b}}$ & 0 & 0 & 0 \\
\hline Medianamente diversificados ${ }^{c}$ & 192 & 175 & 174 \\
\hline Diversificados $^{\mathrm{d}}$ & 111 & 128 & 125 \\
\hline Altamente diversificados ${ }^{\mathrm{e}}$ & 0 & 0 & 5 \\
\hline \multicolumn{4}{|l|}{ Municipios rurales } \\
\hline Muy poco diversificados ${ }^{a}$ & 107 & 105 & 80 \\
\hline Poco diversificados ${ }^{\mathrm{b}}$ & 204 & 241 & 255 \\
\hline Medianamente diversificados ${ }^{c}$ & 1.335 & 1.313 & 1.334 \\
\hline Diversificados $^{\mathrm{d}}$ & 139 & 126 & 114 \\
\hline Altamente diversificados ${ }^{e}$ & 0 & 0 & 2 \\
\hline
\end{tabular}

a) $\mathrm{IS}<\mathrm{E}(\mathrm{IS})-2 \mathrm{ISDT}$

b) $\mathrm{E}(\mathrm{IS})-2 \mathrm{SDT} \leq \mathrm{IS}<\mathrm{E}(\mathrm{IS})-\mathrm{ISDT}$

c) $\mathrm{E}(\mathrm{IS})-\mathrm{I} S \mathrm{~T} \mathrm{~T} \leq \mathrm{IS}<\mathrm{E}(\mathrm{IS})+\mathrm{I} S \mathrm{~T} \mathrm{~T}$

d) $\mathrm{E}(\mathrm{IS})+\mathrm{I} S \mathrm{DT} \leq \mathrm{IS}<\mathrm{E}(\mathrm{IS})+2 \mathrm{SDT}$

e) $\mathrm{E}(\mathrm{IS})+2 \mathrm{SDT} \leq \mathrm{IS}$

DONDE:

IS = VALOR QUE TOMA EL ÍNDICE DE SHANNON

$\mathrm{E}(\mathrm{S})=$ MEDIA DEL IS

SD= DESVIACIÓN ESTÁNDAR DEL IS

$\mathrm{T}=\mathrm{AÑ} O$

FUENTE ELABORACIÓN PROPIA CON DATOS PROCEDENTES DE LOS CENSOS ECONÓMICOS I 999, 2004 Y 2009

\section{Consideraciones finales}

Los servicios son el principal sector empleador en México, aunque la composición al interior de dicho sector se caracteriza por contar con una elevada cantidad de empleos que registran muy baja productividad. En términos espaciales, los servicios se encuentran claramente estructurados de manera jerárquica. Los resultados presentados indican que los servicios con mayor productividad se encuentran localizados, principalmente, en las ciudades de mayor tamańo, siendo la ZMCM la que acapara una mayor cantidad. La incipiente desconcentración de estos se relaciona con la reestructuración espacial de las actividades industriales y turísticas. 
De igual manera, la diversificación económica, vista desde una perspectiva espacial, parece tener una influencia importante en la concentración de ciertas actividades. Sin embargo, por los datos presentados en este artículo, no se puede llegar a conclusiones categóricas. En próximos artículos se debería tomar este punto para analizarlo de manera más exhaustiva.

Conocer con mayor profundidad la distribución espacial de los servicios (tanto en términos de concentración como de diversificación) tiene dos trasfondos. El primero es de corte eminentemente económico, particularmente con lógicas a distintas escalas. En términos globales, implica establecer cómo ciertas regiones se insertan en los mercados internacionales a través de algunas actividades concretas de servicios. Regionalmente, es importante determinar la forma en que se establecen encadenamientos productivos entre distintas actividades; localmente, en tanto, un punto importante para la toma de decisiones se refiere a cuál debería ser la política en materia productiva.

Por otro lado, en términos sociales, el punto central para un país como México es planificar espacialmente la distribución de aquellos servicios sociales que son importantes para superar la pobreza en regiones con grandes carencias. Por ejemplo, lugares centrales para la dotación de servicios como los educativos y los de salud.

Si bien es cierto que este artículo no llegó hasta este último punto, pensamos que es importante iniciar la discusión sobre cuál es la distribución espacial de los servicios, así como cuál debería ser la óptima, tomando en cuenta aspectos económicos y sociales.

\section{Referencias bibliográficas}

Aguilar, A. G. (1999). Mexico City growth and regional dispersal: The expansion of largest cities and new spatial forms. Habitat International, 23(3), 391-412. http://.dx.doi.10.1016/ S0197-3975(99)00014-4

Angoa, I., Pérez-Mendoza, S. \& Polèse, M. (2009). Los tres Méxicos: análisis de la distribución espacial del empleo en la industria y los servicios superiores, por tamaño urbano y por región. EURE, 35(104), 121-143. http://dx.doi.org/10.4067/S025071612009000100006

Banco Mundial (2016). Servicios, etc., valor agregado (\% del PIB). Datos sobre las cuentas nacionales del Banco Mundial y archivos de datos sobre cuentas nacionales de la OCDE. En http://datos.bancomundial.org/indicador/NV.SRV.TETC.ZS

Baumol, W. (1967). Macroeconomics of unbalanced growth: The anatomy of urban crisis. American Economic Review, 57(3), 415-426. http://dx.doi.org/10.2307/1812111

Bettencourt, L., Ostrom, A. L., Brown, S. W. \& Roundtree, R. I. (2002). Client co-production in knowledge-intensive business services. California Management Review, 44(4), 100128. http://dx.doi.10.2307/41166145

Bryson, J. R. \& P. W. Daniels (2015). Handbook of Service Business. Management, marketing, innovation and internalization. Cheltenham, UK: Edward Edgar.

Castells, M. (1977). La cuestión urbana. México, D.F.: Siglo XXI Editores. 
Christaller, W. (1933). Central places in Southern Germany, Englewood Cliffs, NJ: Prentice Hall.

Coffey, W. J. (2000). The geographies of producer services. Urban Geography, 21(2), 170-183. http://dx.doi.org/10.2747/0272-3638.21.2.170

Cuadrado-Roura, J. R. (ed.). (2013a). Service industries and regions. Growth, location and regional effects. Heidelberg and New York: Springer.

Cuadrado-Roura, J. R (2013b). The location of service industries. En J. R. Cuadrado-Roura (ed.), Service industries and regions. Growth, local and regional effects (pp. 253-284). Heidelberg and New York: Springer.

Delgado, B. \& García-Velasco, M. M. (2013). Geographical distributon and regional specialization of KiBs: An empirical investigation across European Region. En J. R . Cuadrado-Roura (ed.), Service industries and regions. Growth, local and regional effects (pp. 305-338). Heidelberg and New York: Springer.

Demarco, M. \& Matusitz, J. (2011). The impact of central place theory on Wal-Mart. Journal of Human Behavior in the Social Enviroment, 21(2), 130-141. http://dx.d oi.10.1080/10911359.2011.542991

Duranton, G. \& Diego Puga (2000). Diversity and specialization in cities: Why, where and when does it matter? Urban Studies, 37(3), 533-555. En http://www.gonzalo.depeco. econo.unlp.edu.ar/economia_aglomeracion/duranton-puga00.pdf

Einchengreen, B. \& Gupta, P. (2013). The two waves of service-sector growth. Oxford Economic Papers, 65(1), 96-123. https://dx.doi.org/10.1093/oep/gpr059

Garza, G. (coord.) (2009). Evolución del sector servicios en ciudades y regiones en México. México, D.F.: El Colegio de México.

Garza, G. (coord.) (2006). La organización espacial del sector servicios en México. México, D.F.: El Colegio de México.

Geppert, K., Gorning, M. \& Werwatz, A. (2008). Economic growth of agglomerations and geographic concentration of industries: Evidence for West Germany. Regional Studies, 42(3), 413-421. http://dx.doi.org/10.1080/00343400701291518

Gervais, A. \& Bradford Jensen, J. (2013). The tradability of services: geographic concentration and trade costs The National Bureau of Economic Research (NBER) Working Paper, 19759. Cambridge, MA: NBER. En http://www.nber.org/papers/w19759 [Versiones previas de este documento circularon con el título "Are services tradable? Evidence from us microdata"]

Gordon, I. R. \& Kaplanis, I. (2014). Accounting for Big-City growth in low paid occupations: immigration and/or service class consumption. Economic Geography, 90(1), 67-90. http://dx.doi.10.1111/ecge.12026

Graizbord, B., Rowland, A. \& Aguilar, A. G. (2003). Mexico city as peripheral global player: The two sides of the coin, Annals of Regional Science, 37(2), 501-518.

Hanssens, H., Derudder, B. \& Witlox, F. (2013). Are advanced producer services connectors for regional economies? An exploration of the geographies of advanced producer services procurement in Belgium. Geoforum, 47(June 2013), 12-21. https://dx.doi. org/10.1016/j.geoforum.2013.02.004

Hermelin, B. (2007). The urbanization and suburbanization of the service economy: Producer services and specialization in Stockholm. Geografiska Annaler. Series B: Human Geography, 89(S1), 59-74. http://dx.doi.10.1111/j.1468-0467.2007.00260.x 
Hsu, W. (2012). Central place theory and city size distribution. Economic Journal, 122(563), 903-930. http://dx.doi 10.1111/j.1468-0297.2012.02518.x

Illeris, S. (2005). The role of services in regional and urban development: A reappraisal of our understanding. The Service Industries Journal, 25(4), 447-460.

Instituto Nacional de Estadística y Geografía (INEGI), México (1999). Censos Económicos, 1999. En http://www.inegi.org.mx/est/contenidos/proyectos/ce/ce1999/

Instituto Nacional de Estadística y Geografía (INEGI), México (2009). Censos Económicos, 2009. En http://www.inegi.org.mx/est/contenidos/espanol/proyectos/censos/ce2009/

Instituto Nacional de Estadística y Geografía (INEGI), México (2004). Censos Económicos, 2004. En http://www.inegi.org.mx/est/contenidos/proyectos/ce/ce2004/

Instituto Nacional de Estadística y Geografía (INEGI), México (2015). Encuesta Nacional de Ocupación y Empleo (enoe), 2015. Aguascalientes: INEGr. En http://www.beta.inegi. org.mx/proyectos/enchogares/regulares/enoe/

Johansson, B. \& Karlss, C. (2009). Knowledge and regional development. En R. Capello \& P. Nijkamp (eds.), Handbook of Regional Growth and Development Theories (pp. 239255). http://dx.doi.org/10.4067/S0250-71612002008500006.

Koch, A. \& Stahlecker, T. (2006). Regional innovation systems and the foundation of knowledge intensive business services. A comparative study in Bremen, Munich, and Stuttgart, Germany. European Planning Studies, 14(2), 123-146. http://dx.doi. org/10.1080/09654310500417830

Merino, F. \& Rubalcaba, L. (2013). Are knowledge-intensive services highly concentrated? Evidence from European regions. Tijdschrift voor Economische en Sociale Geografie, 104(2), 215-232. http://dx.doi.10.1111/j.1467-9663.2012.00739.x

Miller, J. P. \& Bluestone, H. (1988). Prospects for service sector employment growth in nonmetropolitan America. The Review of Regional Studies, 18(1), 28-41. En http://journal. srsa.org/ojs/index.php/RRS/article/viewFile/18.1.5/616

Miles, I., Kastrinos, N. \& Flanagan, Bilderbeek, K., Den Hertog, P., Huntink, W. \& Bouman, M. (1995). Knowledge-intensive business services: Users, carriers and sources of innovation. A report to European Innovation Monitoring System (eIMs) Reports, European Commission DG XIII (sPRINT-EIMs). En https://www.escholar.manchester.ac.uk/uk-acman-scw:75252

Moulaert, F. \& Gallouj, C. (1993). The locational geography of advanced producer service firms: the limits of economies of agglomeration. The Service Industries Journal, 13(2), 91-106. http://dx.doi.org/10.1080/02642069300000032

Mulligan, G., Partridge, M. D. \& Carruthers, J. I. (2012). Central Place theory and its reemergence in regional science. The Annals of the Regional Science, 48(2), 405-431. http://dx.doi.10.1007/s00168-011-0496-7

Nachum, L. (2000). Economic geography and the location of TNCs: Financial and professional service FDI to the USA. Journal of International Business Studies, 31(3), 367-385. http:// dx.doi.10.1057/palgrave.jibs.8490912

Ortiz, E. (2006), El sector servicios en la transformación de la estructura económica de México, 1900-2003. En G. Garza (coord.), La organización especial del sector servicios en México (pp. 79-96). México, D.F.: El Colegio de México.

Parnreirter, C. (2002). Ciudad de México: el camino hacia una ciudad global. EURE, 28(85), 89-119. http://dx.doi.org/10.4067/S0250-71612002008500006 
Parr, J. (2002b). The location of economic activity: Central place theory and the wider urban system. En P. McCann (ed.), Industrial location economics (pp. 32-82). Cheltenham, uK: Edward Elgar Publishing.

Pérez-Campuzano, E. (2010). Segregación socioespacial en una ciudad turística: el caso de Puerto Vallarta, México. Región y Sociedad, 22(49), 143-176. En http://www.scielo. org.mx/pdf/regsoc/v22n49/v22n49a6.pdf

Pérez, E. \& Santos, C. (2013). Tendencias recientes de la migración interna en México. Papeles de Población, 19(76), 53-88. En http://www.scielo.org.mx/scielo.php?script=sci_arttex t\&pid=S1405-74252013000200003

Portes, A. (1995). En torno a la informalidad. Ensayos sobre teoría y medición de la economía no regulada. México, D.F.; Miguel Ángel Porrúa.

Sassen, S. (2001). The global city: New York, London, Tokyo, Princeton, NJ: Princeton University Press.

Searle, G. H. (1998). Changes in producer services location, Sydney: Globalisation, technology and labour. Asia Pacific Viewpoint, 39(2), 237-255. http:// dx.doi.10.1111/1467-8373.00066

Shearmur, R. \& Doloreux, D. (2014). Knowledge-intensive business (KIBS) use and user innovation: High-order services, geographic of hierarchies and internet use in Quebec's manufacturing sector. Regional Studies, 40(10), 1654-1671. http://dx.doi.org/10.1080 /00343404.2013.870988

Shearmur, R. \& Doloreux, D. (2009). Place, space and distance. Towards a geography of knowledge-intensive business services innovation. Industry and Innovation, 6(1), 79102. http://dx.doi.10.1080/13662710902728001

Shearmur, R. \& Doloreux, D. (2008). Urban hierarchy or local buzz? High-order producer service and (or) knowledge-intensive business service location in Canada, 1991-2001. The Professional Geographer, 60(3), 333-355.

Ström, P. (2015). Service research and economic geography. En J. R. Bryson \& P. W. Daniels (eds.), Handbook of Service Business. Management, Marketing, Innovation and Internationalisation. Cheltenham, uK: Edward Elgar Publishing.

Tanaka, H. \& Okamoto, T. (2008). Effects of information communication technology on urban and rural service sectors: An empirical analysis of Japanese Economic Geography. En M. Oya, R. Uda \& Yasunobu (eds.), IFIP International Federation for Information Processing, Towards Sustainable Society on Ubiquitous Networks Vol. 286 (pp. 265-277). Boston: Springer,.

Taylor, P., Derudder, B., Hoyler, M. \& Ni, P. (2013). New regional geographies of the world as practiced by leading advanced producer service firms in 2010. Transactions of the Institute of British Geographers, 38(3), 497-511.

Tickell, A. (1999), The geographies of services: New wine in old bottles. Progress in Human Geography, 23(4), 633-639.

Valdivia, M. (2013). Presencia e impacto especial de los sectores creativos en las zonas metropolitanas de México. Estudios Fronterizos, 15(30), 215-259. http:// dx.doi.10.1111/j.1475-5661.2012.00545.x

Vias, A. (2004). Bigger stores, more stores, or no stores: Paths of retail restructuring in rural America. Journal of Rural Studies, 20(3), 303-318. https://dx.doi.org/10.1016/j. jrurstud.2003.10.003 
Wernerheim, M. \& Sharpe, C. A. (2005). Employment and location patterns of advanced services in non-urban Canada. The Services Industries Journal, 25(2), 181-211. http:// dx.doi.org/10.1080/0264206042000305411

Wood, P. (2006). Urban development and knowledge-intensive business services: too many unanswered questions? Growth and Change, 37(3), 335-361. http://dx.doi.10.1111/ j.1468-2257.2006.00327.x

World Bank (2009). World Development Report 2009: Reshaping economic geography. Washington, D.c.: The World Bank. En https://openknowledge.worldbank.org/ handle/10986/5991 